\title{
Resonance-shifting Integral Resonant Control for High-speed Nanopositioning
}

\author{
Sumeet S. Aphale ${ }^{1}$ and Mohammad Namavar ${ }^{1}$ and Andrew J. Fleming ${ }^{2}$
}

\begin{abstract}
The first resonance mode of mechanical systems is a significant limit to the achievable positioning bandwidth. This resonance is dependent on the physical, material and geometric properties of the system. Significant effort is typically required to increase the resonance frequency by increasing stiffness or reducing mass. In this article, a modified IRC scheme is presented that effectively shifts the first resonance mode to a higher frequency, thereby enabling a substantially higher positioning bandwidth. A $70 \%$ increase in positioning bandwidth is demonstrated.
\end{abstract}

\section{INTRODUCTION}

Integral Resonant Control (IRC) was first proposed, a decade ago, as a simple, low-order well-performing damping control scheme for co-located systems, [1]. The advantages of the IRC scheme include the ability to damp multiple resonant modes, guaranteed stability and robustness in the presence of resonance frequency shifts - an artefact of system loading. These desirable qualities have made the IRC scheme a popular choice for resonance damping applications. As a result, it has been successfully applied to damp the problematic resonances in a plethora of technological systems such as cantilever beams [1], flexible robotic manipulators [2], piezoelectric-stack actuated nanopositioners [3], piezoelectric tube nanopositioners [4], piezoelectric microgrippers [5], lightweight pedestrian structures [6], floor structures [7], nonlinear oscillatory systems [8], MEMS nanopositioners [9] etc. In a number of these applications, the IRC scheme is used as the damping controller in tandem with a suitably designed tracking controller to effectively deliver superior positioning performance.

It is well-known that most micro- and nanopositioning systems exhibit a lightly damped resonant mode at a relatively low frequency. Moreover, this resonant mode stringently restricts the achievable closed-loop positioning bandwidth of the positioning system, [10], [11]. To increase the achievable positioning bandwidth, several geometries and structural design modifications have been proposed that place this limiting first resonant mode at higher frequencies, [3], [12], [13]. Unfortunately, this increase in bandwidth by means of a higher first resonance frequency comes at the cost of a reduction in displacement ranges. It is therefore deemed extremely beneficial if the achievable positioning bandwidth could be increased without any mechanical / design changes; thereby keeping the displacement range unchanged. A way to achieve this using standard control techniques has hitherto not been reported.

\footnotetext{
${ }^{1}$ Center for Applied Dynamics Research, School of Engineering, University of Aberdeen, UK. s.aphale@abdn.ac.uk

${ }^{2}$ Precision Mechatronics Lab, University of Newcastle, Callaghan, NSW, Australia. www.pmtxlab.com/peple
}

In this work, the first successful implementation of a control scheme that effectively increases the positioning bandwidth of a nanopositioning system by seemingly shifting the dominant first resonant mode to a higher frequency, is reported. A proportional gain controller is implemented in tandem with the appropriate IRC scheme to result in a closed-loop damped system that has its first resonance shifted to a higher frequency than it's open-loop counterpart. This technique can be easily integrated into any co-located positioning system to deliver a significantly wider positioning bandwidth. As a result, the proposed scheme has the potential to significantly increase achievable scan speeds in Atomic Force Microscopes.

\section{A. Overview}

In section II, a second-order model (for the dominant first resonant mode) is identified to match the measured frequency response of one axis of the nanopositioning platform. A brief introduction for the IRC design is also included. Section III presents the rationale, the detailed design and closed-loop performance analysis of the proposed resonance-shifting method. Section IV presents the modifications necessary to implement the resonance-shifting IRC scheme in practice. Positioning performance of the closed-loop resonance-shifted IRC scheme is recorded and presented in both time and frequency domain to show that significant increase in positioning bandwidth is achieved. VI concludes the paper.

\section{System Modeling}

A simplified schematic of one axis of a piezoelectric-stack actuated nanopositioner is shown in Fig. 1. The piezoelectric actuator is fixed at one end to a base and at the other end, it pushed on a compliant structure which acts as a lever motion amplifier. The motion amplifier transfers the motion to the moving stage which is supported by flexure joints to ensure maximal flatness, low cross-coupling and linearity of motion. The motion of the moving stage is accurately sensed by a capacitive displacement sensor.

The frequency response of one axis of a nanopositioner measured from the voltage applied to the piezo-stack to the resultant displacement of the platform as sensed by the capacitive displacement sensor can be represented as an infinite sum of second-order resonant sections shown in Eq. 1.

$$
G(s)=\sum_{i=1}^{M} \frac{\sigma_{i}^{2}}{s^{2}+2 \zeta_{i} \omega_{i} s+\omega_{i}^{2}}
$$


Piezoelectric actuator

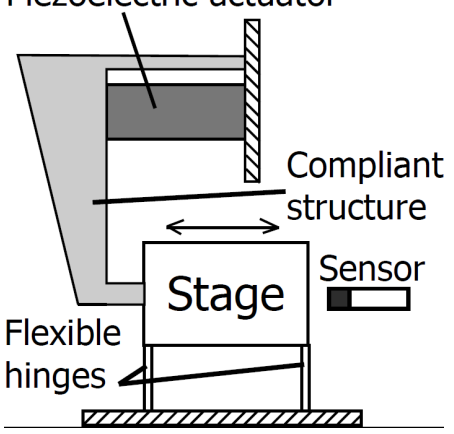

Fig. 1. Schematic view of the mechanical structure of the nanopositioner axis and displacement sensor arrangement.

where $M \rightarrow \infty, \sigma_{i}^{2}$ corresponds to the gain of each mode of vibration, $\zeta_{i}$ is the damping ratio of each mode, and $\omega_{i}$ is is the natural frequency of vibration of each mode. However, for practical purposes Eq. (1) is usually truncated to a contain finite number modes that lie within the bandwidth of interest. For typical nanopositioners, the first resonant mode is usually very lightly damped and dominates the entire frequency response of the axis. Consequently, in most related works, the axis model is truncated to include only the first dominant resonant mode [12], [14], [15]. This dominant lightly damped resonance mode can be modeled as a second order system with a small and positive feed-through term $d$, added to compensate for the truncationinduced modeling error, [16]. Thus, the useable model of one axis of a typical nanopositioner takes the form:

$$
G(s)=\frac{\sigma^{2}}{s^{2}+2 \zeta \omega_{p} s+\omega_{p}^{2}}+d,
$$

where $\zeta$ is the damping coefficient and $\omega_{p}$ is the resonance frequency.

The frequency-response of the nanopositioning platform was measured from input voltage supplied to the piezo-actuator to output voltage proportional to the displacement measured by the capacitive displacement sensor. A subspace-based identification technique as reported in [17], was employed to generate a second-order model shown in Eq. 3

$$
\hat{G(s)}=\frac{8.3782 \times 10^{6}}{s^{2}+57.2 s+6.657 \times 10^{6}} .
$$

The measured frequency response data as well as the identified second-order model's frequency response is plotted in Fig. 2. As seen, the identified model matches the measured data with good accuracy. The first resonant mode at $411 \mathrm{~Hz}$ is lightly damped and dominates the dynamics withing the bandwidth of interest. As an artifact of the mechanical construction of the platform axis, a second resonant mode, much smaller than the dominant first mode can be seen at $576 \mathrm{~Hz}$. Though this mode is within the bandwidth of interest, simulation results show that neglecting it from the system model and subsequent control design and analysis does not have any unwanted effects.

In the next section, this identified model is used to derive
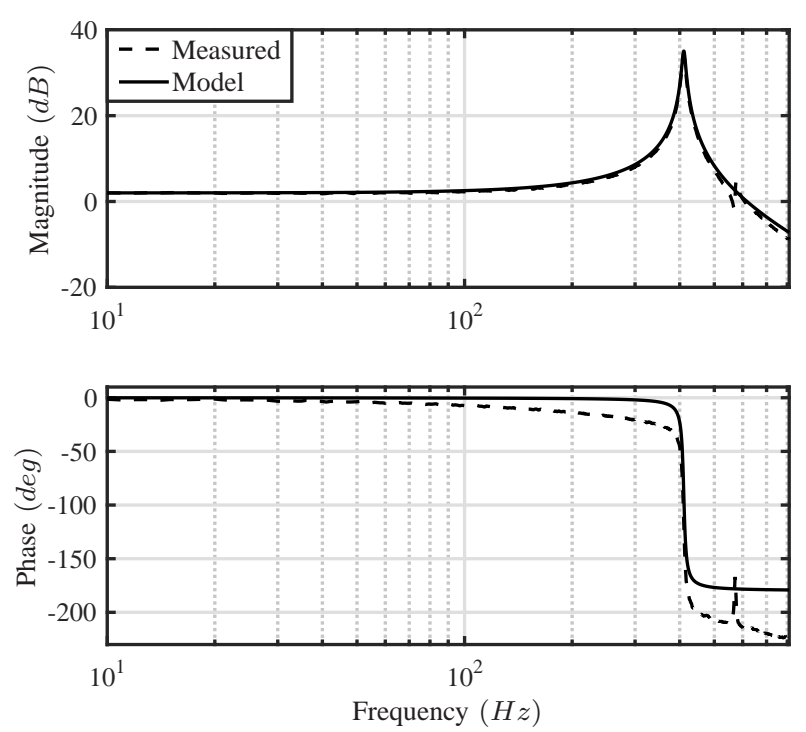

Fig. 2. Measured FRF vs the identified second-order model's frequency response

the controller parameters as well as to validate the designed control scheme via simulations.

\section{Controller Design}

The three step controller design process is described as follows:

\section{Step 1: Resonance shifting -}

The achievable positioning bandwidth of a nanopositioner is limited by the resonance frequency of its axis. The resonanceshifting controller is introduced to overcome this constraint. This controller is basically a negative proportional feedback loop, see Fig. 3.

The proportional gain loop not only increases the resonance frequency but also increases the maximum peak value (by reducing the damping coefficient $\zeta$ ). This negative feedback loop is always stable for a second order system and by increasing the gain $\hat{k}$ the DC gain of the loop approaches unity. This is shown below where $H_{1}$ is the transfer function for the resonance-shifted closed-loop system:

$$
\begin{aligned}
\hat{G} & =\frac{\sigma^{2}}{s^{2}+2 \zeta \omega_{p} s+\omega_{p}^{2}} \\
H_{1} & =\frac{\hat{k} G}{1+\hat{k} G} \\
H_{1} & =\frac{\hat{k} \sigma^{2}}{s^{2}+2 \zeta \omega_{p} s+\omega_{p}^{2}+\hat{k} \sigma^{2}}
\end{aligned}
$$

Henceforth, the system subjected to damping and tracking controller is given by:

$$
H_{1}(s)=\frac{\bar{\sigma}^{2}}{s^{2}+2 \bar{\zeta} \bar{\omega}_{p} s+\bar{\omega}_{p}^{2}}
$$




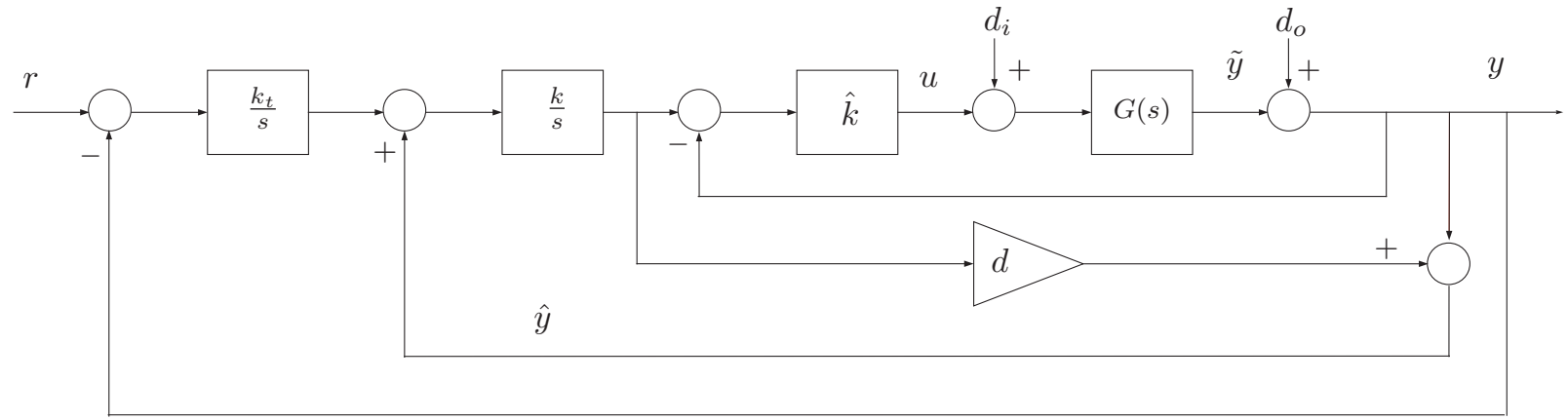

Fig. 3. (a) Block diagram for the Resonance-shifting controller, IRC damping controller and Integral tracking controller scheme where $d$ is the feed-through term, $\hat{k}$ is the resonance shifting gain, $k$ is the IRC damping gain and $k_{t}$ is the integral tracking gain.

where $\bar{\sigma}^{2}=\hat{k} \sigma^{2}$ and $\bar{\omega}_{p}^{2}=\omega_{p}^{2}+\hat{k} \sigma^{2}$.

By comparing the platform transfer function and $H_{1}$, relationships between $\bar{\zeta}$ and other parameters can be identified:

$$
\begin{array}{r}
2 \bar{\zeta} \bar{\omega}_{p}=2 \zeta \omega_{p} \\
\Rightarrow \frac{\bar{\zeta}}{\zeta}=\frac{\omega_{p}}{\bar{\omega}_{p}}
\end{array}
$$

(5) shows that increasing resonance frequency by a factor of $\alpha$ will reduce the damping ratio by $1 / \alpha$. In most closedloop second-order system analysis, the damping ratio is usually neglected for sake of simplicity. The same simplification will be applied to all the analysis presented in the work that follows. As we have further reduced the damping coefficient, this simplification is more valid. The DC gain of $H_{1}$ is $\hat{k} \sigma^{2} /\left(\omega_{p}^{2}+\hat{k} \sigma^{2}\right)$. Since for most of the systems $\sigma^{2}$ and $\omega_{p}^{2}$ are almost equal, the DC gain can be estimated as:

$$
D C_{\text {gain }} \approx \frac{\hat{k} \omega_{p}^{2}}{(\hat{k}+1) \omega_{p}^{2}} \approx 1=0 d B
$$

1) Relation between sensor bandwidth and $\hat{k}$ : Sensor bandwidth is one of the limiting factors for the amount of resonance shift. The shifted resonance should be within the sensor bandwidth $\gamma$. Considering shifted resonance frequency be $\left(\omega_{p}^{2}+\hat{k} \sigma^{2}\right)^{1 / 2}$, then:

$$
\begin{array}{r}
\left(\omega_{p}^{2}+\hat{k} \sigma^{2}\right)^{1 / 2}<\gamma \\
\hat{k}<\frac{\gamma^{2}-\omega_{p}^{2}}{\sigma^{2}}
\end{array}
$$

Step 2: Damping Controller - The general concept of the IRC design can summarized below:

Given that a colocated system $G(s)$ with pole-zero interlacing is to be damped, an adequate feed-through term ' $d$ ' can first be added to the system to reverse the interlacing from pole-zero to zero-pole. Furthermore, if a simple integrator $C(s)=\frac{k}{s}$ is implemented in positive feedback with such a modified system $\hat{G}(s)=G(s)+d$, as the integral gain ' $k$ ' is increased, the poles of the system traverse a curve where first they move away from the imaginary axis, into the left-half complex plane (thus increasing their damping coefficient) and then back towards the imaginary axis till they reach their correspondingly paired zero (with reduced damping).

2) Relationship between feed-through, damping and IRC gain: In [18], a full mathematical derivation of the relationship between feed-through, damping and IRC gain can be summarized by the following theorem.

Theorem 1: Consider a colocated system with a pair of complex poles at $\pm j \omega_{p}$ and feed-through induced zeros at $j \omega_{z}>j \omega_{p} / 3$. If the IRC strategy is implemented, the maximum damping achievable is given by

$$
\zeta_{\max }=\frac{1}{2}\left(\frac{\omega_{p}}{\sqrt{\omega_{p}^{2}+\sigma^{2} / d}}-1\right) .
$$

The controller gain required to reach this maximum damping is given by

$$
k=\frac{1}{|d|}\left(\omega_{p} \sqrt{\frac{\omega_{p}}{\sqrt{\omega_{p}^{2}+\sigma^{2} / d}}}\right),
$$

where $\omega_{z}=\sqrt{\omega_{p}^{2}+\sigma^{2} / d}$ with respect to $d$.

Step 3: Tracking controller - The IRC algorithm has been applied to damp the resonances of various precision positioning systems, especially nanopositioners [2], [3], [6], [14], [19]. Nanopositioning systems generally employ piezoelectric actuators that tend to introduce nonlinear effects such as hysteresis and creep. To minimize the positioning errors introduced by these phenomena, a damping controller such as IRC is used in conjunction with a simple integral tracking scheme [10], [20]. A block diagram of the complete control scheme incorporating both IRC damping and integral tracking along with the resonance-shifting controller is shown in Fig. (3).

In Fig. (3), the transfer functions of interest for quantification of positioning performance are $y / r$ (output to input) and $y / d_{i}$ (output to input disturbance). To find the characteristic equation 
$C(s)$, four loops $L_{1}, L_{2}, L_{3}$ and $L_{4}$ are defined as below:

$$
\begin{aligned}
L_{1} & =-\hat{k} \times G(s) \\
L_{2} & =\frac{k}{s} \times \hat{k} \times G(s) \\
L_{3} & =\frac{k}{s} \times d \\
L_{4} & =-\frac{k_{t}}{s} \times \frac{k}{s} \times \hat{k} \times G(s) .
\end{aligned}
$$

Using Mason's rule, $C(s)$ is the numerator of (9).

$$
1-\left(L_{1}+L_{2}+L_{3}+L_{4}\right)+\left(L_{1} L_{3}\right)
$$

Roots of the denominator of (9) are zeros of different transfer functions or they may cancel out. But the zeros of (9) are the poles of the final closed-loop transfer function. Stability of the closed-loop transfer function is the most important factor in any control application. $H_{1}$ as defined before, can be considered as the new system which needs to be damped and tracked. Hence, the relationship between damping and tracking controller can be defined by Theorem 2, [18].

Theorem 2: Let $k$ and $k_{t}$ be the IRC damping and integral tracking gains respectively. Then, for the closed-loop system as implemented in Fig. (3) to be stable, the gains must obey the following inequality:

$$
k_{t} k<-\frac{\bar{\sigma}^{2}+d \bar{\omega}_{p}^{2}}{d^{2}}
$$

This theorem proves that damping and tracking gains are related in the IRC scheme and cannot be arbitrarily tuned independent of each other.

Corollary 3: For a given second order system controlled using the scheme shown in Fig. (3), there exists an absolute maximum value for $k_{t} k$. The corresponding maximum value is related to $d$ by:

$$
\begin{gathered}
d=-2 \frac{\bar{\sigma}^{2}}{\bar{\omega}_{p}^{2}}=2 d_{c} \\
\max \left\{k_{t} k\right\}=\frac{\bar{\omega}_{p}^{4}}{4 \bar{\sigma}^{2}} \\
\text { IV. EXPERIMENTS }
\end{gathered}
$$

Experimental validation is carried out by implementing the developed control scheme on a nanopositioning platform. Straightforward implementation leads to an unstable closedloop system. Practical implementation of this technique needs a modified control structure. The following section will first describe the issue and then present the modified implementable control structure.

\section{A. Modified implementation for practical application}

The control block diagram illustrated in Figure 3 cannot be implemented directly due to the wide bandwidth of the proportional feedback loop. The unlimited bandwidth can cause instability when dealing with unmodeled high frequency

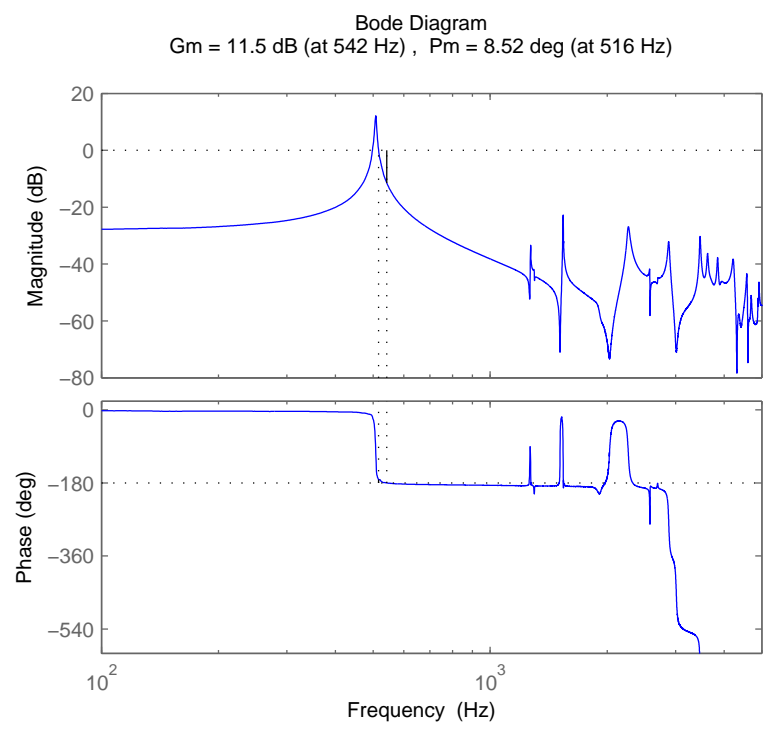

Fig. 4. The loop-gain and stability margins of the proportional feedback loop when $k_{s}=0.02$.

modes. The practical stability margins is best determined directly from the measured open-loop frequency response. With this approach, the maximum stable gain of the proportional feedback was found to be $k_{s}=0.02$ which is only sufficient to achieve an $8 \mathrm{~Hz}$ increase in the resonance frequency. The loop gain in Figure 4 shows a phase margin of 8 degrees, which is of little practical value.

Although the proportional loop is not practical in isolation, the characteristics are significantly more favorable when the proportional and damping control loops are combined.

First, the complementary sensitivity function of the proportional loop is

$$
G_{s}(s)=\frac{G_{s}(s) k_{s}}{1+G_{s}(s) k_{s}} .
$$

Similarly, the complementary sensitivity of the damping control loop is

$$
G_{d}(s)=\frac{G_{s}(s) C_{d}(s)}{1-G_{s}(s) C_{d}(s)},
$$

where $C_{d}(s)=k_{d} / s$. By substituting $G_{s}(s)$, the transfer function of the damping and proportional loop is

$$
\begin{aligned}
G_{d}(s) & =\frac{G(s) C_{d}(s) k_{s}}{1+G(s) k_{s}-G(s) C_{d}(s) k_{s}}, \\
G_{d}(s) & =\frac{G(s) C_{d}(s) k_{s}}{1+G(s) k_{s}\left(1-C_{d}(s)\right)} .
\end{aligned}
$$

By removing the factor $C_{d}(s) /\left(1-C_{d}(s)\right)$ from the above equation, the transfer function can be arranged in the form of a regulator and prefilter. That is,

$$
G_{d}(s)=C_{f}(s) \frac{G(s) C_{e q}(s)}{1+G(s) C_{e q}(s)},
$$




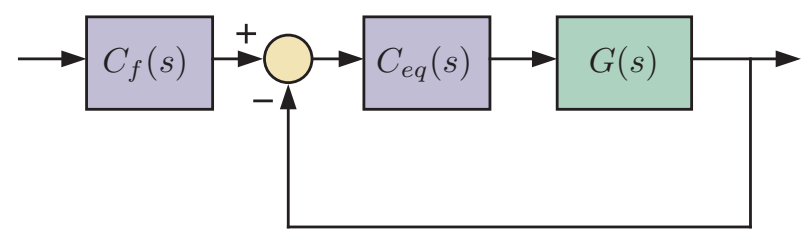

Fig. 5. Equivalent regulator form of the proportional and damping loops.

where the equivalent regulator is

$$
C_{e q}(s)=k_{s}\left(1-C_{d}(s)\right)
$$

and the prefilter is

$$
C_{f}(s)=\frac{C_{d}(s)}{1-C_{d}(s)},
$$

This arrangement is illustrated in Figure 5. Although there are other possible implementations, this configuration is desirable since the prefilter and regulator are both causal and stable. In contrast, standard IRC requires an unstable controller or the use of positive feedback. Furthermore, the stability margins of the equivalent control loop are superior to the isolated proportional loop, as described in the following section.

\section{B. Experimental Results}

A proportional gain of $k_{s}=1.5$ was chosen the increase the resonance frequency from $507 \mathrm{~Hz}$ to $1000 \mathrm{~Hz}$. The optimal damping parameters were then determined to be $k_{d}=6000$ and $d=-1.2$. That is, the equivalent regulator is

$$
C_{e q}(s)=\frac{1.5 s+1800}{s+7200},
$$

and the prefilter is

$$
C_{f}(s)=\frac{6000 s+4.32 \times 10^{7}}{s^{2}+8400 s+8.64 \times 10^{6}} .
$$

The loop-gain and stability margins of the equivalent damping control loop are plotted in Figure 6. Even though the proportional gain has been increased by two orders of magnitude, the phase margin is significantly better than the isolated proportional loop.

The frequency responses of the standard and resonance shifted damping control loops are plotted in Figure 7. Both controllers eliminate the resonance peak; however, the resonance shifted controller achieves a bandwidth almost twice that of the standard controller and nearly double the open-loop resonance frequency.

The servo controller was tuned to minimize settling time after a step command. The servo controllers for the standard IRC $C_{1}(s)$ and resonance shifted systems $C_{2}(s)$ were found to be

$$
C_{1}(s)=\frac{650}{s}+0.034, \text { and } C_{2}(s)=\frac{840}{s}+0.034
$$

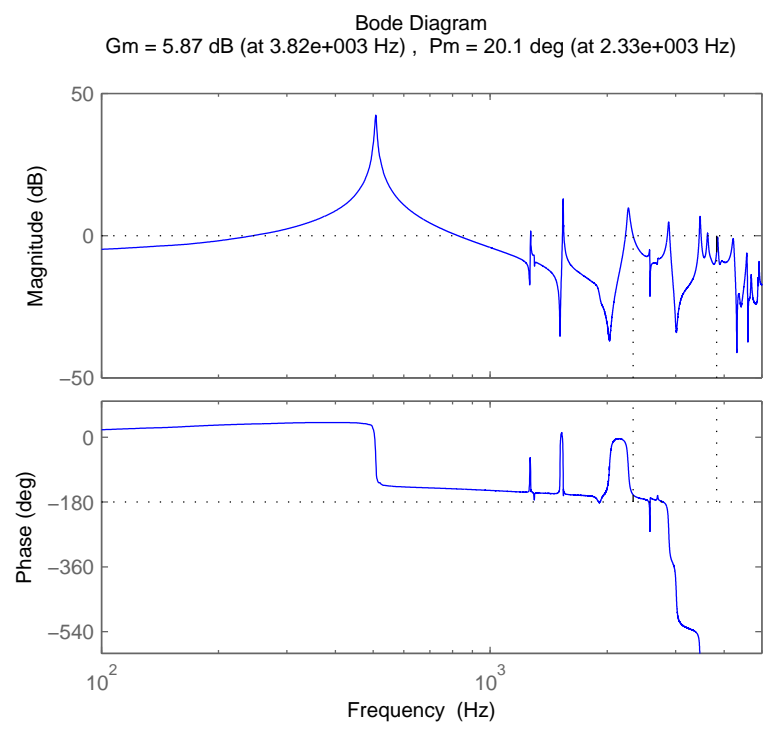

Fig. 6. The loop-gain and stability margins of the equivalent damping control loop.

The closed-loop frequency responses of the servo loops are compared in Figure 8. The resonance shifted controller permits an increase in the tracking bandwidth from $225 \mathrm{~Hz}$ to $711 \mathrm{~Hz}$, which is $40 \%$ higher than the system resonance. This is an outstanding achievement for a first-order damping and tracking controller that does not require model based inversion.

The system responses are also compared in the time domain in Figures 9, 10, 11 and 12. The increased bandwidth of the resonance shifted controller permits a faster settling time and lower tracking error for dynamic signals.

Remarks: The resonance shifted controller was demonstrated to effectively double the resonance frequency of the nanopositioner whilst increasing the damping to near critical level. Since the resonance frequency of a mechanical system is proportional to $\sqrt{k / m}$, a comparable increase using mechanical methods would require a four times increase in the stiffness, or a $75 \%$ reduction in the mass. Such mechanical improvements may be difficult, undesirable, or impossible to achieve. Therefore, the proposed technique provides an alternate or complementary method to significantly improve the performance without additional cost or mechanical reconfiguration.

\section{ANALOG IMPLEMENTATION}

Due to the low order of the control scheme, it is straightforward to implement in both digital and analog forms. There are a number of options for analog implementation of Figure 5. One method is to implement the transfer functions (20) and (21) directly, for example, by state-variable filter. However, this approach may be difficult to tune experimentally. It is preferable to create circuits with component values that are directly related to the control gains.

In order to implement Figure 5 as an analog controller, circuits are required for the transfer functions $C_{e q}(s)$ and $C_{f}(s)$. Both of these filters are parameterized by the damping 

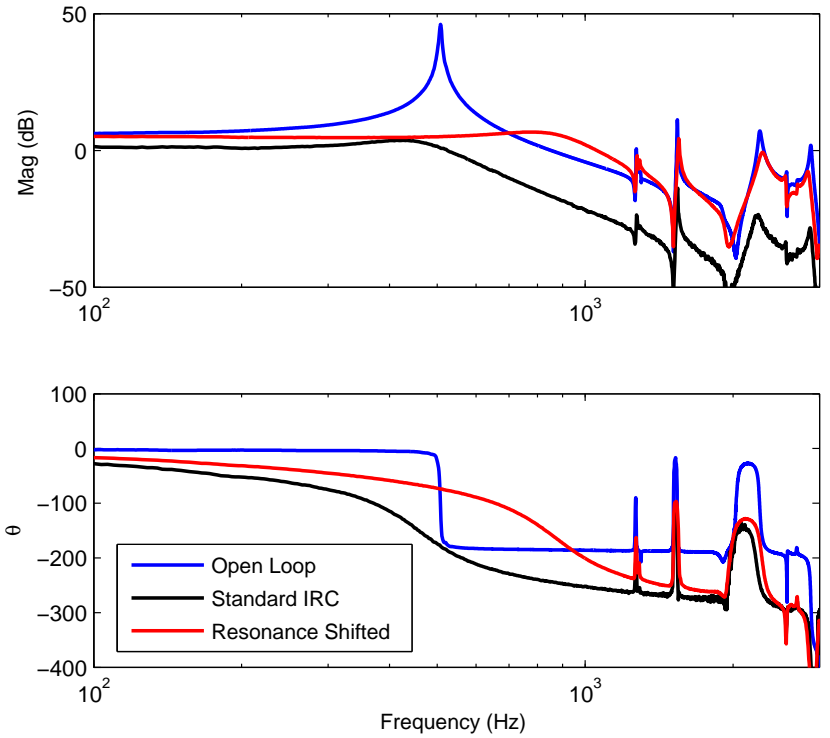

Fig. 7. Experimental frequency response of the damping loop (in $\mu \mathrm{m} / \mathrm{V}$ ) The resonance frequency of the open-loop system is $508 \mathrm{~Hz}$ while the $3 \mathrm{~dB}$ bandwidth of the standard IRC and resonance shifted controllers is $565 \mathrm{~Hz}$ and $1002 \mathrm{~Hz}$
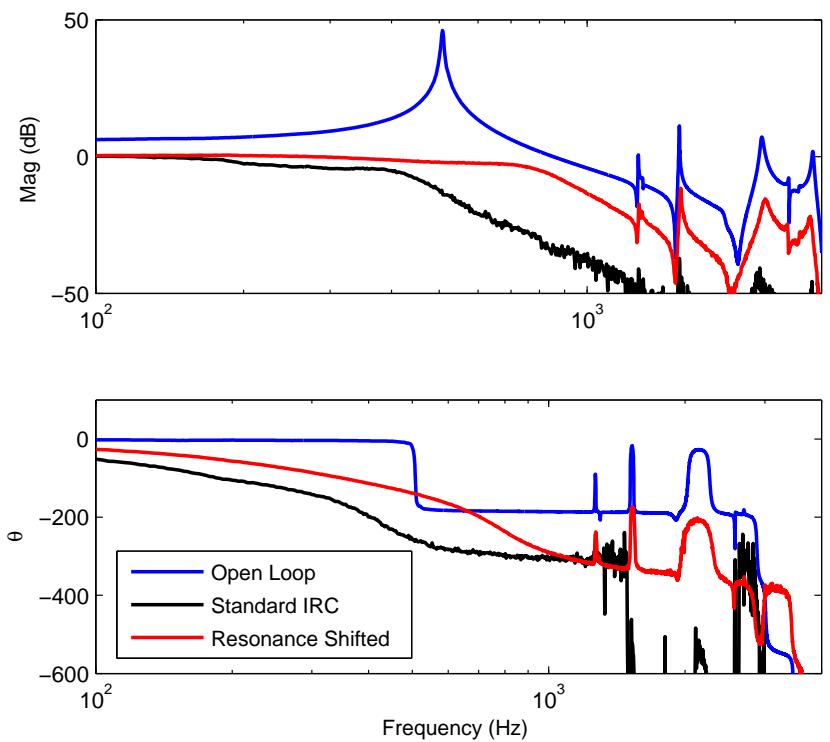

Fig. 8. Experimental frequency response of the complete servo loop (in $\mu \mathrm{m} / \mathrm{V}$ ). The $3 \mathrm{~dB}$ bandwidth of the IRC control system is $225 \mathrm{~Hz}$ while the resonance shifted controller is $711 \mathrm{~Hz}, 40 \%$ higher than the resonance frequency.

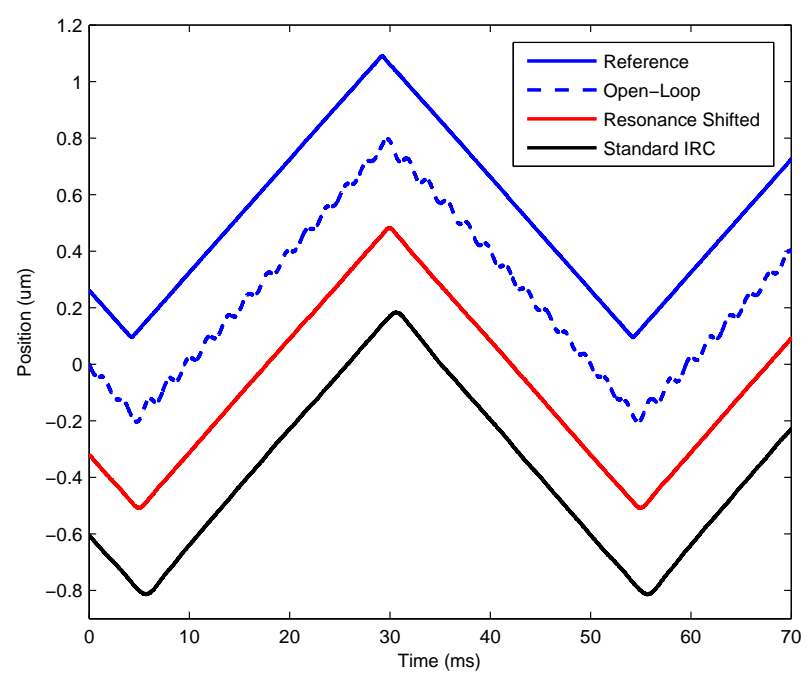

Fig. 9. Servo responses to a $20-\mathrm{Hz}$ triangle wave.

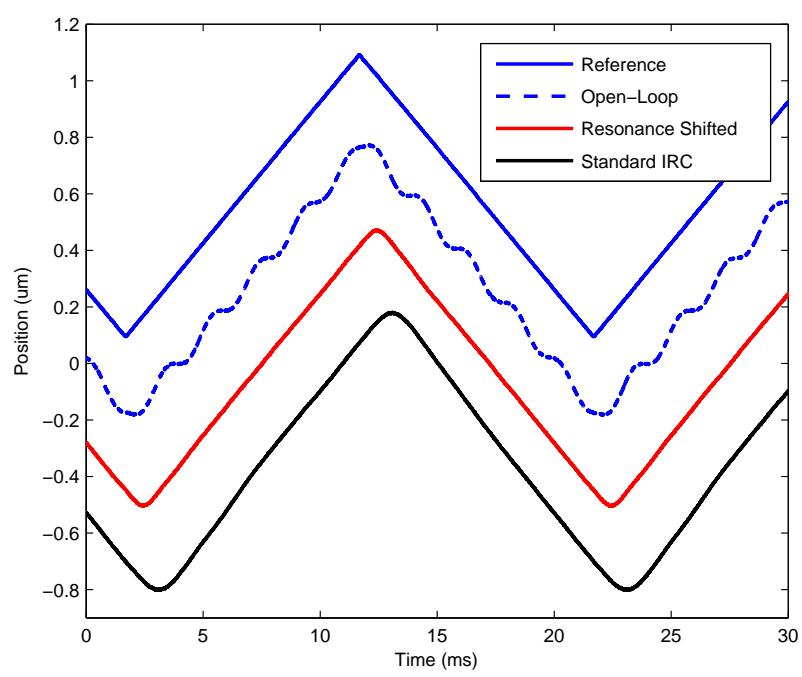

Fig. 10. Servo response to a $50-\mathrm{Hz}$ triangle wave.

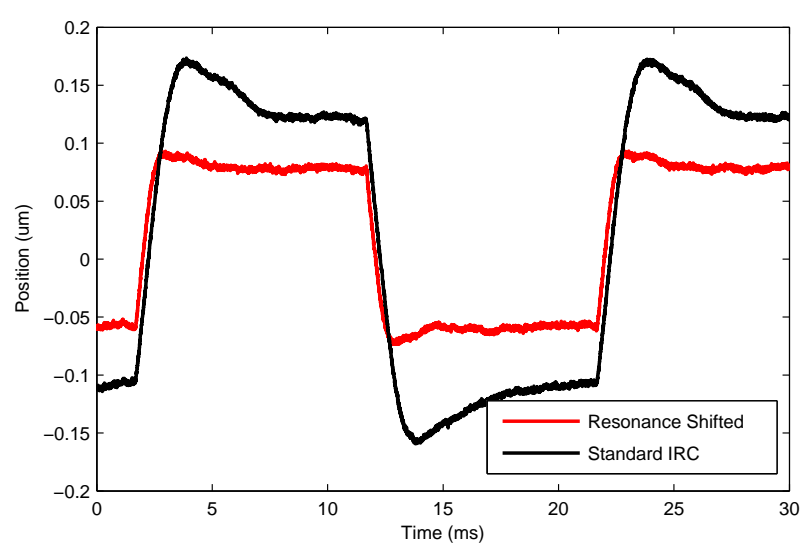

Fig. 11. Servo error for the $50-\mathrm{Hz}$ triangle wave. 


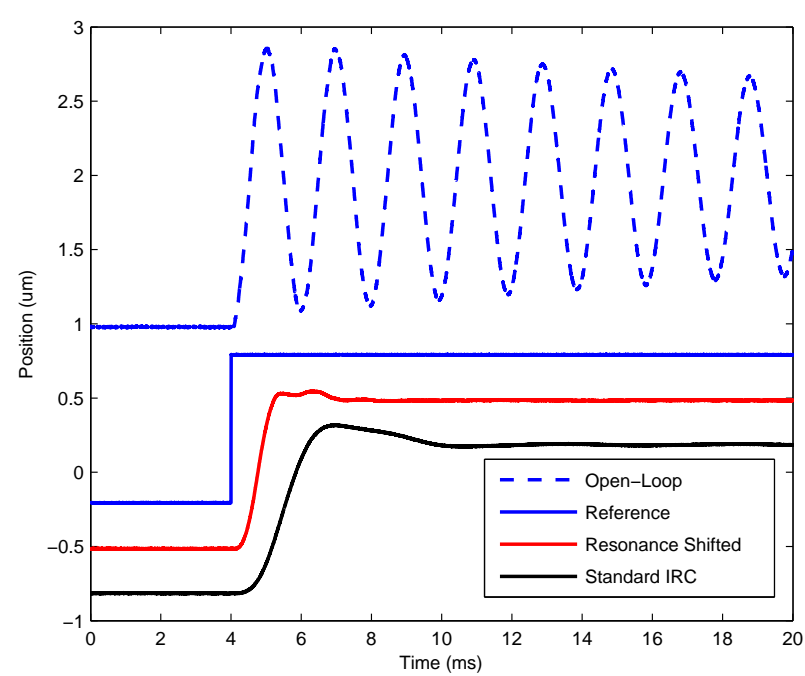

Fig. 12. Servo response to a step input.

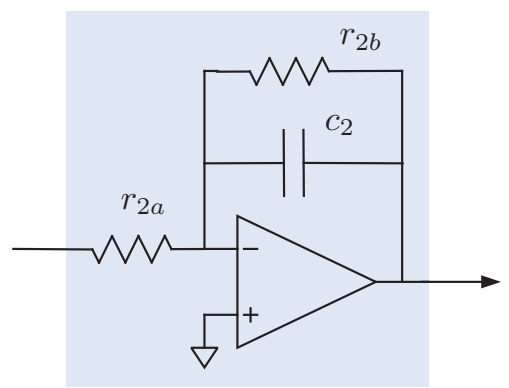

Fig. 13. Analog implementation of the IRC damping controller $-C_{d}(s)$.

controller $C_{d}(s)$. A filter that implements $-C_{d}(s)$ is illustrated in Figure 13. The desired transfer function is

$$
-C_{d}(s)=-\frac{k_{d}}{s-d k_{d}} .
$$

The circuit transfer function is

$$
\frac{-\frac{1}{r_{2 a} c_{2}}}{s+\frac{1}{r_{2 b} c_{2}}} .
$$

Since $k_{d}$ is positive and $d$ is negative, the equalities are

$$
r_{2 a} c_{2}=\frac{1}{k_{d}}, \text { and } r_{2 b} c_{2}=\frac{1}{d k_{d}} \text {. }
$$

The transfer function $C_{e q}(s)$ can be constructed from $-C_{d}(s)$ as illustrated in Figure 14.

The transfer function $C_{f}(s)$ can be implemented by noticing that the sub-circuit $-C_{d}(s)$ is contained in a unity-gain negative feedback loop with an inverting input gain. This arrangement can be implemented by the circuit in Figure 15.

With circuit diagrams for $C_{e q}(s)$ and $C_{f}(s)$, the block diagram in Figure 5 can be implemented with the addition of a subtracter. Once the damping and resonance control loop has been implemented, it is trivial to implement the outer tracking control loop with standard circuits.

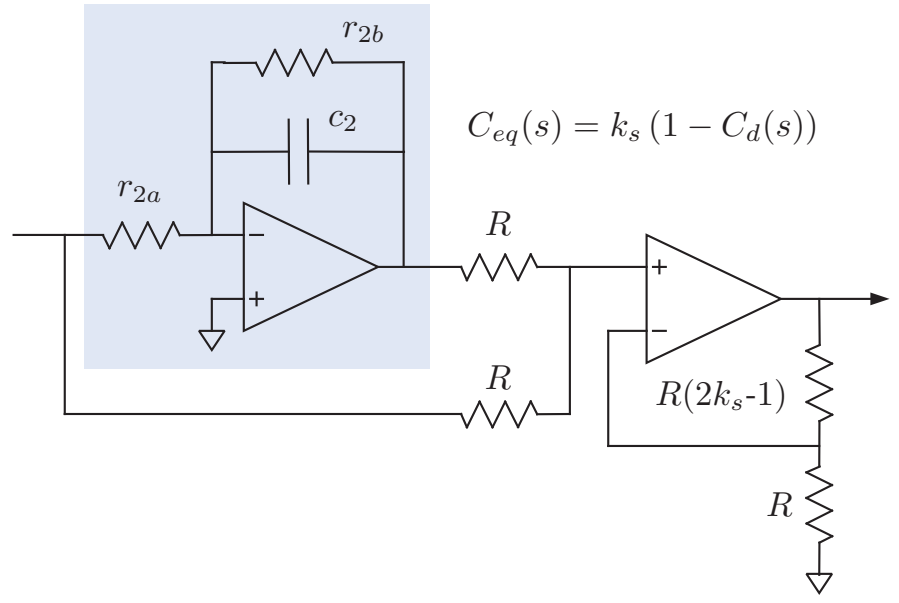

Fig. 14. Analog implementation of $C_{e q}(s)$. The sub-circuit for $-C_{d}(s)$ is shaded.

$$
C_{f}(s)=C_{d}(s) /\left(1-C_{d}(s)\right)
$$

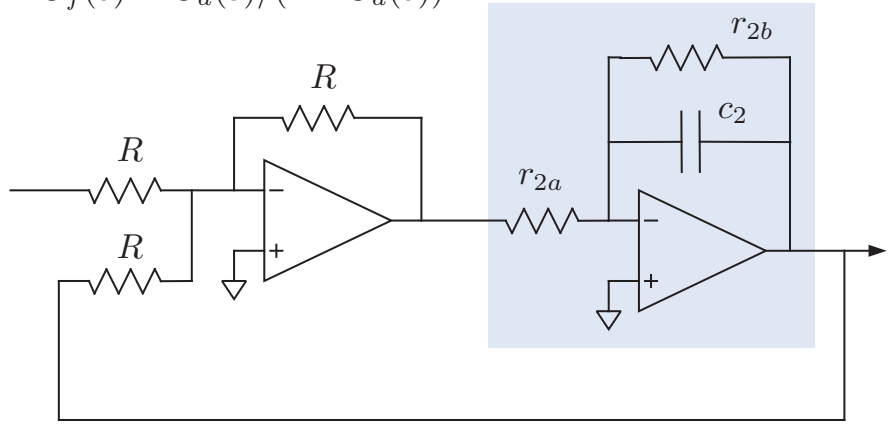

Fig. 15. Analog implementation of $C_{f}(s)$. The sub-circuit for $-C_{d}(s)$ is shaded.

\section{CONCLUSIONS}

In this work, the IRC scheme is modified to include a resonance shifting control loop that is capable of increasing the positioning bandwidth of any colocated nanopositioner. The modified scheme also possesses excellent input disturbance rejection capability - a key performance criteria for accurate positioning systems. Finally, the effectiveness of the proposed scheme is demonstrated by simulating the resonanceshifting IRC scheme on the model derived from an FRF data set recorded for a commercially available nanopositioner. A twelve-fold increase in positioning bandwidth was achieved. Future work will include experimental verification and bandwidth-dictated parameter optimizations.

\section{REFERENCES}

[1] S. S. Aphale, A. J. Fleming, and S. O. R. Moheimani, "Integral resonant rontrol of collocated smart sturctures," Smart Materials and Structures, vol. 16, pp. 439 - 446, 2007.

[2] E. Pereira, S. S. Aphale, V. Feliu, and S. O. R. Moheimani, "Integral resonant control for vibration damping and precise tip-positioning of a single-link flexible manipulator," IEEE/ASME Transactions on Mechatronics, vol. 16, no. 2, pp. 232 - 240, April 2011. 
[3] Y. K. Yong, S. S. Aphale, and S. O. R. Moheimani, "Design, identification, and control of a flexure-based xy stage for fast nanoscale positioning," I EEE Transactions on Nanotechnology, vol. 8, no. 1, pp. 45 - 54, January 2009.

[4] B. Bhikkaji and S. O. R. Moheimani, "Integral resonant control of a piezoelectric tube actuator for fast nanoscale positioning," IEEE/ASME Transactions on Mechatronics, vol. 13, no. 5, pp. 530 - 537, October 2008.

[5] M. Grossard, M. Boukallel, N. Chaillet, and C. Rotinat-Libersa, "Modeling and robust control strategy for a control-optimized piezoelectric microgripper," IEEE/ASME Transactions on Mechatronics, vol. 16 , no. 4, pp. 674 - 683, August 2011.

[6] I. M. Diaz, E. Pereira, and P. Reynolds, "Integral resonant control scheme for cancelling human?induced vibrations in light?weight pedestrian structures," Structural Control and Health Monitoring, vol. 19, no. 1, pp. 55 - 69, February 2012.

[7] M. J. Hudson and P. Reynolds, "Implementation considerations for active vibration control in the design of floor structures," Engineering Structures, vol. 44, pp. 334 - 358, November 2012.

[8] E. Omidi and S. N. Mahmoodi, "Sensitivity analysis of the nonlinear integral positive position feedback and integral resonant controllers on vibration suppression of nonlinear oscillatory systems," Communications in Nonlinear Science and Numerical Simulation, vol. 22, no. 1-3, pp. 149 - 166, mAY 2015.

[9] M. Maroufi, A. Bazaei, and S. O. R. Moheimani, "A high-bandwidth mems nanopositioner for on-chip afm: Design, characterization, and control," IEEE Transactions on Control Systems Technology, vol. 23, no. 2, pp. $504-512$, March 2015.

[10] S. Devasia, E. Eleftheriou, and S. Moheimani, "A survey of control issues in nanopositioning," IEEE, vol. 15, no. 5, pp. 802 - 823, 2007.

[11] A. A. Eielsen, M. Vagia, J. T. Gravdahl, and K. Y. Pettersen, "Damping and tracking control schemes for nanopositioning," IEEE/ASME Transactions on Mechatronics, vol. 19, no. 2, pp. 432 - 444, April 2014.

[12] Y. K. Yong, B. Bhikkaji, and S. O. R. Moheimani, "Design, modeling, and fpaa-based control of a high-speed atomic force microscope nanopositioner," IEEE/ASME Transactions on Mechatronics, vol. 18, no. 3 , pp. 1060 - 1071, June 2013.

[13] A. P. Nievergelt, B. W. Erickson, N. Hosseini, J. D. Adams, and G. E. Fantnera, "Studying biological membranes with extended range high-speed atomic force microscopy," Scientific Reports, vol. 6, p. doi: 10.1038/srep21654, 2015.

[14] A. J. Fleming, S. S. Aphale, and S. O. R. Moheimani, "A new method for robust damping and tracking control of scanning probe microscope positioning stages," IEEE Transactions on Nanotechnology, vol. 9, no. 4, pp. 438 - 448, 2010.

[15] D. Russell, A. J. Fleming, and S. S. Aphale, "Simultaneous optimization of damping and tracking controller parameters via selective pole placement for enhanced positioning bandwidth of nanopositioners," Journal of Dynamic Systems, Measurement, and Control, vol. 137, no. 10, p. 8, 2015 .

[16] R. L. Clark, "Accounting for out-of-bandwidth modes in the assumed modes approach: Implications on colocated output feedback control,' Journal of Dynamic Systems, Measurement and Control, Transactions of the ASME, vol. 119, no. 3, pp. 390-395, 1997.

[17] T. McKelvey, H. Akay, and L. Ljung, "Subspace-based multivariable system identification from frequency response data," IEEE Transactions on Automatic Control, vol. 41, no. 7, pp. 960-979, 1996.

[18] M. Namavar, A. J. Fleming, M. Aleyaasin, K. Nakkeeran, and S. S. Aphale, "An analytical approach to integral resonant control of secondorder systems," IEEE/ASME Transactions on Mechatronics, vol. 19, no. 2, pp. $651-659,2014$.

[19] B. Basu and S. R. K. Nielsen, "A multi-modal control using a hybrid pole-placementintegral resonant controller (ppir) with experimental investigations," Structural Control and Health Monitoring, vol. 18, pp. 191 206, 2011.

[20] S. S. Aphale, B. Bhikkaji, and S. O. R. Moheimani, "Minimizing scanning errors in piezoelectric stack-actuated nanopositioning platforms," IEEE Trans. on Nanotechnology, vol. 7, no. 9, pp. 79 - 90, 2008. 